\title{
Aircraft trajectory simulator using a three degrees of freedom aircraft point mass model
}

\author{
Javier García-Heras Carretero $\neq$, Francisco Javier Sáez Nieto †, Ricardo Román Cordón * \\ Air Navigation Research Group \\ Polytechnic University of Madrid \\ Madrid, Spain \\ ‡ javier.garciaheras@upm.es, $\uparrow$ franciscojavier.saez@upm.es, + ricardo.roman@upm.es
}

\begin{abstract}
Aircraft Operators Companies (AOCs) are always willing to keep the cost of a flight as low as possible. These costs could be modelled using a function of the fuel consumption, time of flight and fixed cost (over flight cost, maintenance, etc.). These are strongly dependant on the atmospheric conditions, the presence of winds and the aircraft performance. For this reason, much research effort is being put in the development of numerical and graphical techniques for defining the optimal trajectory.

This paper presents a different approach to accommodate AOCs preferences, adding value to their activities, through the development of a tool, called aircraft trajectory simulator. This tool is able to simulate the actual flight of an aircraft with the constraints imposed. The simulator is based on a point mass model of the aircraft.

The aim of this paper is to evaluate $3 \mathrm{DoF}$ aircraft model errors with BADA data through real data from Flight Data Recorder FDR. Therefore, to validate the proposed simulation tool a comparative analysis of the state variables vector is made between an actual flight and the same flight using the simulator. Finally, an example of a cruise phase is presented, where a conventional levelled flight is compared with a continuous climb flight. The comparison results show the potential benefits of following user-preferred routes for commercial flights.
\end{abstract}

\section{Author Keywords \\ Trajectory Based Operation; Optimal Control; Business Trajectory; Point Mass Model; Trajectory Optimization; Aircraft Model Validation}

\section{ACM Classification Keywords} H.5.m.

\section{INTRODUCTION}

Over the past few years, the common practice within ATM has been that commercial aircraft must fly by following a set

Permission to make digital or hard copies of all or part of this work for personal or classroom use is granted without fee provided that copies are not made or distributed for profit or commercial advantage and that copies bear this notice and the full citation on the first page. To copy otherwise, or republish, to post on servers or to redistribute to lists, requires prior specific permission and/or a fee. of predefined routes to reach their destinations. Currently, aircraft operators (AOCs) are requesting for more flexibility to fly according to their preferences, in order to help them achieve their business objectives. AOCs generally wish to keep the cost of a flight as low as possible. These costs depend mainly on: the amount of fuel needed; the actual time of flight and also the over flight charges.

The present paper shows a different approach to accommodate AOCs preferences and add value to their activities, through the development of a tool/algorithm that calculates optimal trajectories.

To accomplish this objective, an aircraft trajectory simulator (TS) based on 3DoF is presented. This model considers the aircraft as a point mass, as well as the aircraft performances and physical constraints, and also atmospheric information. Many works on aircraft trajectory modelling or trajectory optimization have used a $3 \mathrm{DoF}$ aircraft model, as an example $[1,2,3,4,5]$.

In order to accept the model as an aircraft TS, an adequate validation is needed. According to [6] a validation process involves: the evaluation of the software used, the airframe and the pilot. This paper is focused on the software and aircraft model validation. To evaluate the accuracy of the model, it could be developed from an experimental flight test to an aircraft observation during several parameters are modified.

For this reason, the uncertainties of the model should be delimited. The data used to perform the validation of the model is obtained from actual flights, and it allows checking the differences between the real variables (from real Flight Data Recorder FDR) and the ones defined by the aircraft TS. A similar method has been used by [7], where an Unmanned Aerial Vehicles (UAV) model based on a $6 \mathrm{DoF}$ has been validated through the comparison at the same inputs of the real aircraft response and the software model one. Also, in [8] a flight test program was designed to validate a $6 \mathrm{DoF}$ flight model.

Once the validation of the aircraft TS has been accomplished an example is presented. In this example we compare two different trajectories: one representing a cruise phase of a real flight following a levelled flight in three steps and the second one performing a continuous climb. 


\section{AICRAFT TRAJECTORY SIMULATOR}

The movement of an aircraft could be expressed by a 6 Degrees of Freedom (6DoF) or a $3 \mathrm{DoF}$ [9]. The first model is the most complete model due to the fact that it has into consideration both rotational and translational motion.

Commercial aircraft trajectories involve small aircraft rotation axes and also the angle of sideslip could be considered negligible because of the turn coordinator system that is installed into almost the whole commercial aircraft. The resultant of that approximation is the 3DOF model.

Therefore, this paper proposes an aircraft TS based on a Point Mass Model (PMM) due to it is a simple one and the errors of that model are affordable, as it will be shown in the follow part. Equation (1) shows in the three first elements the kinematic equations, the following three the dynamic equations and the final element is the fuel consumption equation.

$$
\frac{d}{d t}\left[\begin{array}{l}
X \\
Y \\
h \\
V \\
\psi \\
\gamma \\
W
\end{array}\right]=\left[\begin{array}{l}
V \cos (\psi) \cos (\gamma)+\omega_{x} \\
V \sin (\psi) \cos (\gamma)+\omega_{y} \\
V \sin (\gamma)+\omega_{z} \\
\frac{g}{W}[(T \cos (\theta-\gamma)-D)-W \sin (\gamma)] \\
\frac{g \sin (\phi)}{W V}[L+T \sin (\alpha)] \\
\frac{g}{W V}[(L+T \sin (\theta-\gamma)) \cos (\phi)-W \cos (\gamma)] \\
-C T
\end{array}\right]
$$

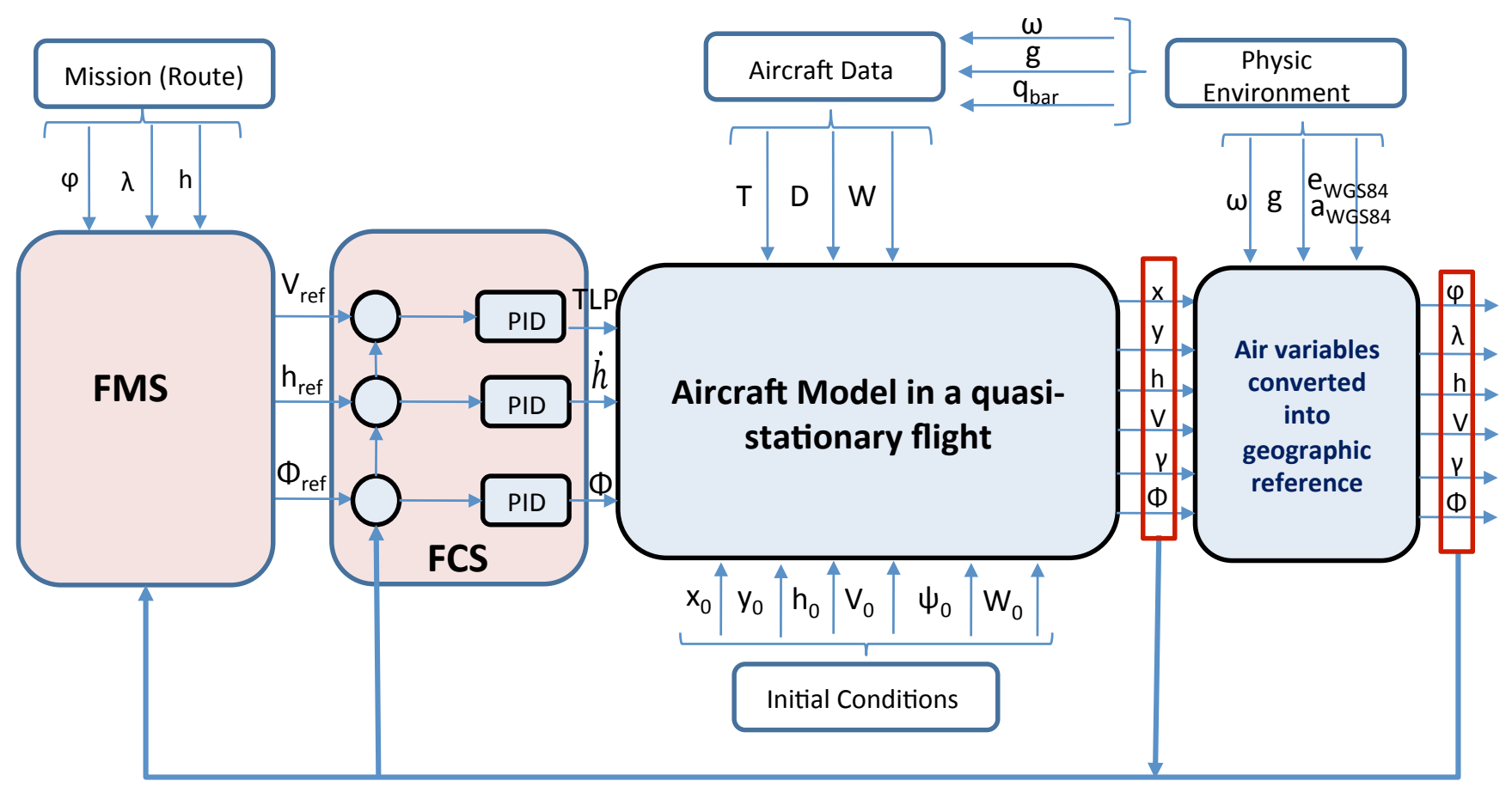

Where:

- $\mathrm{C}$ and $\mathrm{T}$, are the specific fuel consumption and the engine thrust respectively, determined using BADA 3.9 information [10]

- $\mathrm{W}$ is the weight

- $\gamma$ is the flight path angle

- $\mathrm{g}$ is the gravity of value $9.81 \mathrm{~m} / \mathrm{s}^{2}$

- $\mathrm{V}$ is the velocity of the airplane relative to the air

- $\alpha$ is the angle of attack

- $\beta$ is the angle of sideslip

- $\theta, \phi, \psi$ are the airplane pitch, roll and heading angles respectively

- $\mathrm{X}, \mathrm{Y}, \mathrm{h}$ are the component of the position vector along $\mathrm{XYZ}$

- $\omega_{\mathrm{x}}, \omega_{\mathrm{y}}, \omega_{\mathrm{z}}$ are the component of the wind vector along $\mathrm{XYZ}$

- $\mathrm{L}$ is the aircraft lift force, which is considered equal to $W / \cos (\phi)$

- $\mathrm{D}$ is the aircraft drag force, which is evaluated in each phase of flight with drag polar coefficients from BADA 3.9 [10]

Figure 1: Aircraft Trajectory Simulator Scheme. 
Equation (1) could be simplified because in commercial aircraft, the roll angle, the flight path angle and the flight path angle derivative are small. Also, it must be observed that the thrust has been considered as a vector pointing in the longitudinal aircraft axe direction. With all these considerations, the equation (1) could be rewritten as it is shown in (2).

For the design and implementation of the aircraft TS, MATLAB $^{\circledR}$ SIMULINK $^{\circledR}$ [11] software environment was used.

The aircraft TS has four main sections: Flight Management System (FMS), Flight Control System (FCS), aircraft model in a quasi-stationary flight and a geographic variables converter, as is depicted in figure 1.

The FMS manages the reference variables (Velocity, altitude and roll angle) which inputs of the FCS. For this purpose information from the mission (route $2 \mathrm{D} / 3 \mathrm{D}$ or $4 \mathrm{D}$ ) or the aircraft guidance law are needed.

The FCS is based on three control loops to regulate: velocity deviation, altitude deviation and lateral deviation from the defined mission. These control loops will act on Throttle Lever Position (TLP), altitude derivative and roll angle, respectively, which are the inputs in the aircraft model.

In the Aircraft model in a quasi-stationary flight section the kinematics, dynamics and the fuel consumption equation are implemented (see equation 2). It is also taken into account the quasi-stationary hypothesis where lift is considered equal to the weight component. That section needs, besides the inputs, the aircraft performances, power plant information and aircraft initial condition.

$$
\frac{d}{d t}\left[\begin{array}{l}
X \\
Y \\
V \\
\psi \\
W
\end{array}\right]=\left[\begin{array}{l}
V \cdot \cos (\psi)+\omega_{x} \\
V \cdot \sin (\psi)+\omega_{y} \\
V \cdot \gamma+\omega_{z} \\
\frac{g}{W}[T-D-W \cdot \gamma] \\
\frac{g L \cdot \sin (\phi)}{W \cdot V} \\
-C \cdot T
\end{array}\right]
$$

Finally, the coordinates should be converted from Local Level System (LLS) to Latitude, Longitude, and Height (LLH) coordinates with respect to the same system of coordinate. This will allow in further works the analysis of a group of trajectories and their possible interactions within the airspace. For this porpoise, the eccentricity and the mayor axis of the ellipsoid WGS-84 are needed.

\section{VALIDATION AICRAFT TRAJECTORY SIMULATOR}

The validation process is based on the comparison between real data from Flight Data Recorder (FDR) and variables obtained from the aircraft TS. For this purpose, the FDR route is introduced into the Mission section of the aircraft TS (see Figure 1). The information of the FDR selected for this validation example corresponds to a Iberia flight IB6826 from São Paulo - Guarulhos $\left(23^{\circ} 25^{\prime} 55^{\prime \prime} \mathrm{S} 46^{\circ} 28^{\prime} 10^{\prime \prime} \mathrm{W}\right)$ to Madrid - Barajas $\left(40^{\circ} 29^{\prime} 36.80^{\prime \prime} \mathrm{N} 3^{\circ} 34^{\prime} 0.035^{\prime \prime} \mathrm{W}\right)$ flown on the $14^{\text {th }}$ of September 2009 (ETOT 19:00 local time). This flight lasted 9 hours, 58 minutes and 21 seconds (i.e. 35901 seconds) and was flown by an Airbus A340-600.
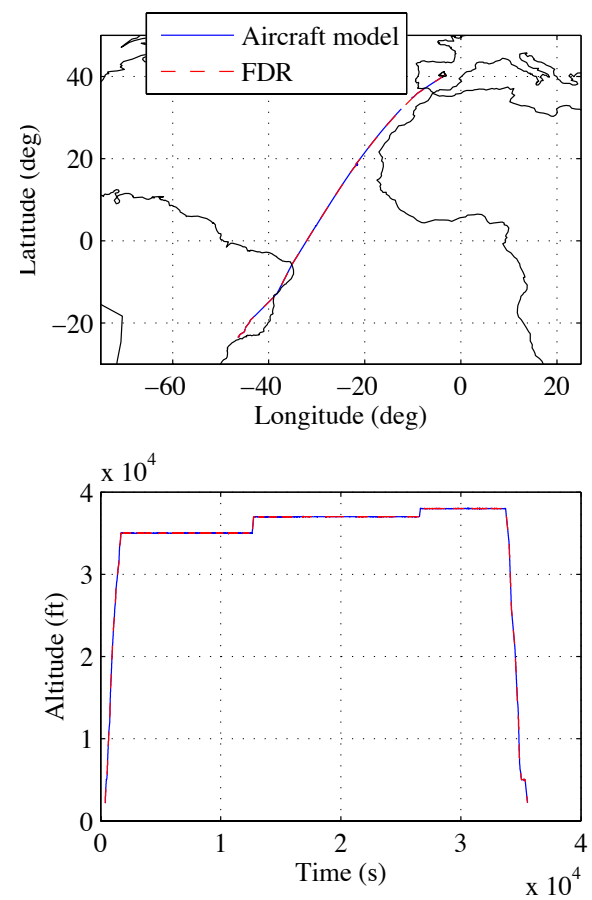

Figure 2. FDR and Aircraft model trajectory

In Figure 2 the trajectory obtained by the aircraft TS (blue line) is compared with the actual trajectory that was flown (red line-FRD) with respect to global coordinates (longitude and latitude). The vertical trajectories of the TS and the actual flight are also compared with respect to time in Figure 2. These comparisons are made for the three phases in which this flight was performed.

The deviation between both trajectories is presented numerically in Table 1, showing these results, the trajectory defined by the aircraft TS can be considered accurate enough to represent a real flight, except for the weight variable witch have shown great deviations. This could be due to the winds uncertainties that have not been considered.

\begin{tabular}{|c|c|c|c|c|c|c|}
\hline & \multicolumn{2}{|c|}{ Take-off } & \multicolumn{2}{c|}{ En-Route } & \multicolumn{2}{c|}{ Landing } \\
\cline { 2 - 7 } & $\boldsymbol{\mu}$ & $\boldsymbol{\sigma}$ & $\boldsymbol{\mu}$ & $\boldsymbol{\sigma}$ & $\boldsymbol{\mu}$ & $\boldsymbol{\sigma}$ \\
\hline $\mathbf{h}$ (ft) & 0.0072 & 36.634 & $5.49 \mathrm{e}-5$ & 1.8951 & 0.0124 & 8.7721 \\
\hline $\begin{array}{c}\mathbf{V} \\
\text { (kts) }\end{array}$ & -2.779 & 73.384 & -0.325 & 1.3033 & 2.9334 & 40.272 \\
\hline $\begin{array}{c}\boldsymbol{\phi} \\
(\mathbf{d e g})\end{array}$ & -0.008 & 106.67 & -0.849 & 49.9047 & 0.9879 & 53.368 \\
\hline $\begin{array}{c}\boldsymbol{\gamma} \\
\mathbf{d e g}\end{array}$ & 0.1985 & 0.7414 & 0.0007 & 0.006868 & 0.0586 & 0.0883 \\
\hline $\begin{array}{c}\mathbf{W} \\
\mathbf{( k g )}\end{array}$ & 1205.6 & 317607 & 1150.8 & 151332.8 & -202.9 & 84216 \\
\hline
\end{tabular}

Table 1: Mean $(\mu)$ and Variance $(\sigma)$ differences for every phase of flight 


\section{PRACTICAL OPTIMIZATION EXAMPLE}

Once the aircraft TS has been validated as an adequate tool for obtaining the aircraft variables along the defined route, a practical example could be presented. In this section the Aircraft TS is used for comparing a flight cruise phase based on three steps (which have been used in the real flight presented in the validation process, see figure 2) and a flight based on a continuous cruise phase. The results obtained with this example allow measuring the quantity of fuel that is saved with the latter using the same time of simulation and a constant True Air Speed (TAS) of 480kts.
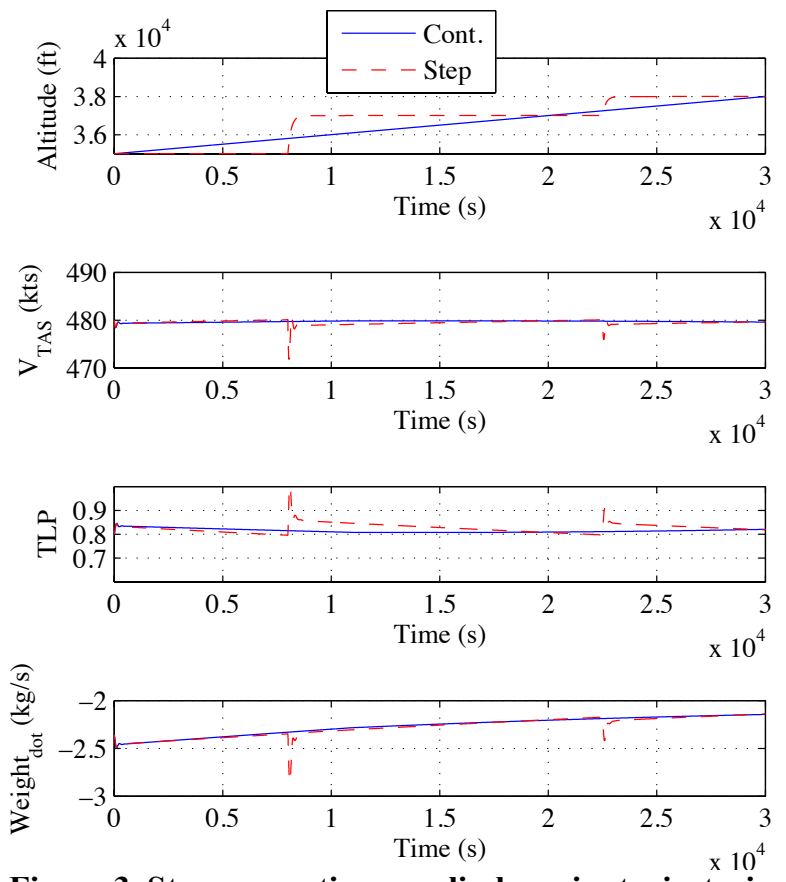

Figure 3. Step vs. continuous climb cruise trajectories

The figure 3 shows the vertical profiles, the TAS, the TLP and the weight derivative of the both trajectories. The TAS is close $480 \mathrm{kts}$ except in the trajectory based on steps when the aircraft changes the flight level, which is the same time of a peak of consumption or a maximum TLP.

In conclusion, the cruise based on steps spend $351.4104 \mathrm{~kg}$ more fuel than the cruise base on continuous climb, because the cruise based on a continuous climb uses more constant TLP than the other one. The more constant use of TLP is associated to constant fuel consumption and besides the optimal aircraft altitude has to be increased due to the waist of mass associate to the fuel consumption.

\section{CONCLUSION AND FURTHER WORKS}

In the present research it is shown that the most simple aircraft model (3DoF in a quasi-stationary flight) can simulate with accuracy the aircraft trajectory taking into account the computational advantages (less processing time) that it gives to the demanded problem. That is important because in future works it will be required to study in a simulated airspace the optimised Business Trajectories (BTs) provided by the Aircraft TS presented on this paper.
The goal of this second activity will be minimize the probability of ATC tactical intervention. It will receive all the Shared Business Trajectories (SBTs), display them, and use an algorithm to evaluate the possible interactions and reduce them to an admissible minimum. In case there are problematic SBTs, i.e. SBTs that are involved in interactions with hazards, they are taking through the whole process again with the new constraints, producing perturbed SBTs, until they tally with the agreed SBT. The agreed SBT are finally adopted by the system as Reference Business Trajectory (RBT).

Moreover, a simple optimization example is presented. A sign of how important is keep studying the trajectory optimization is that only making a continuous climb in the cruise phase a decreasing of fuel consumption is obtained. Therefore, the future objective is to design full optimal 4D trajectories: take-off, climb, cruise, descent and landing phases. Wind must be taken into account in future works.

\section{REFERENCES}

1. Soler M., Olivares A., and Staffetti E., Hybrid Optimal Control Approach to Commercial Aircraft Trajectory Planning, Journal of Guidance, Control, and Dynamics, Vol. 33, No. 3, May-June 2010.

2. Valenzuela A., Aircraft trajectory optimization using parametric optimization theory, PhD thesis, University of Seville, 2012.

3. Akhtar N, Whidborne J F, and Cooke A K, Real-time optimal techniques for unmanned air vehicles fuel saving, Proceedings of the Institution of Mechanical Engineers, Part G: Journal of Aerospace Engineering, , 2011

4. Dharki S., Aircraft flight path optimization the Hamilton-Jacobi-Belman considerations. Applied Mathematical Sciences, Vol. 6, no. 25, 1221-1249, 2012.

5. Howe-Veenstra R., Commercial aircraft trajectory optimization and efficiency of air traffic control procedures, Master's thesis, University of MINNESOTA, 2011.

6. McGovern, S.M., Categories for classification of aircraft flight model validation, Digital Avionics Systems Conference, 2007. DASC '07. IEEE/AIAA 26th, vol., no., pp.6.D.1-1,6.D.1-9, 21-25 Oct. 2007.

7. Chen X.Q., Ou Q., Wong D. R., Li Y. J., Sinclair M. and Marburg A., Flight Dynamics Modelling and Experimental Validation for Unmanned Aerial Vehicles, Mobile Robots - State of the Art in Land, Sea, Air, and Collaborative Missions, XiaoQiChen (Ed.), 2009. XiaoQiChen (Ed.), ISBN: 978-953-307-001-8, InTech, Available

from:http://www.intechopen.com/books/mobile-robotsstate-of-the-art-in-land-sea-air-and- 
collaborativemissions/flight-dynamics-modelling-andexperimental-validation-for-unmanned-aerial-vehicles

8. Ong S.Y., Yeo D., and Gui A., Aircraft six-degrees-offreedom model validation though flight test, $A I A A$ Modeling and Simulation Technologies Conference and Exhibit, Boston MA USA, August 10, 1998.

9. Hull D. H., Fundamental of airplane flight mechanics. Springer-Verlag Berlin and Heidelberg GmbH \& Co. K. 2007.
10. Nuic A., User Manual for the base of Aircraft Data (BADA) Revision 3.9, Eurocontrol Experimental Center, 2008.

11. The Mathworks, Inc. MathWorks España -MATLAB and simulink for technical computing. 1994. Natick, MA: The Mathworks, Inc. http://www.mathworks.es/index.html (1994, accessed March 2013). 